Historia y comunicación social

ISSN-e 1988-3056

https://dx.doi.org/10.5209/hics.66310

\title{
El caso Dreyfus: Intelectuales y prensa española de 1898
}

\author{
Sara Fuentes Garzón ${ }^{1}$
}

Recibido: 2 de agosto de 2017. / Aceptado: 30 de junio de 2018.

Resumen. La desmedida complejidad del asunto Dreyfus a finales del siglo XIX provocó un mayor interés social, la creciente participación de la opinión pública internacional y la consecuente determinación de los intelectuales por defender, criticar o colaborar en la causa. Con el fin de comprender la repercusión que suscitó en España, este artículo tiene como objetivo analizar la actividad periodística de los diarios madrileños La Correspondencia de España, El Liberal y El Imparcial en cuanto espacios de divulgación sobre la cuestión Dreyfus y del pensamiento intelectual de 1898.

Palabras clave: Caso Dreyfus; Historia del periodismo español; El Imparcial; El Liberal; La Correspondencia de España; Émile Zola.

\section{[en] The Dreyfus affair: Intellectuals and Spanish press in 1898}

\begin{abstract}
The complexity of the Dreyfus affair at the end of the $19^{\text {th }}$ century produced a great social interest and the involvement of international public opinion. Moreover, intellectuals took part in the determination to defend, criticize or collaborate in the cause. In order to understand the repercussion in Spain, this article aims to define the activity of the Madrid newspapers El Liberal, La Correspondencia de España and El Imparcial as spaces of promotion on the Dreyfus question and intellectual thought in 1898 .
\end{abstract}

Keywords: Dreyfus affair; History of Spanish journalism; El Imparcial; El Liberal; La Correspondencia de España; Émile Zola.

Sumario: 1. Introducción. 2. Seguimiento periodístico durante el año 1898. 3. Ubicación de la información en cada periódico. 4. Evolución de los titulares. 5. Principales fuentes de información. 6. Conclusiones. 7. Notas. 8. Referencias bibliográficas.

Cómo citar: Fuentes Garzón, S. (2019). El caso Dreyfus: Intelectuales y prensa española de 1898, en Historia y comunicación social 24 (2), 713-726.

\footnotetext{
Universidad Complutense de Madrid. sfuent01@ucm.es
} 


\section{Introducción}

La compleja situación de España en la última década del siglo XIX, impregnada por el gran descontento de su sociedad y una aguda crisis política y económica, generó un contexto hostil en el que intervino un joven grupo de intelectuales (Villacorta, 1980) capaces, por primera vez en la historia, de animar a la opinión pública y ayudar a superar la aguda crisis de credibilidad y de legitimidad manifiestas. Identificados como un nuevo agente social, la "Generación del 98" se preocupó por el futuro de la nación (Chabás, 2001: 4), participó en el ámbito político, debido a su compromiso intrínseco con la sociedad, y encontró un espacio de difusión de su pensamiento en los diarios españoles (Fuentes y Fernández, 2010: 171). La prensa española distaba del modelo de prensa de masas de finales del siglo XIX (Saiz y Seoane, 1998: 2728), impulsado en Gran Bretaña, en Francia y en Estados Unidos. Sin embargo, se iniciaba de forma paulatina el cambio hacia el modelo de periódico de empresa (Barrera y Sánchez, 1992: 204-206). La Correspondencia de España (Madrid, 1859), El Liberal (Madrid, 1879) y El Imparcial (Madrid, 1867) entraban en competencia, al ser los periódicos generalistas de mayor tirada, y comenzaron a utilizar titulares de gran tamaño y a impulsar secciones con cierto atractivo para el público lector.

El periodo seleccionado para este estudio es el año 1898. La sociedad española contaba con un índice de analfabetismo muy elevado, en el último tercio del siglo XIX alcanzaba en torno al $72 \%$ de la población siendo el $81 \%$ mujeres (Tuñón, 2000: 95). Esta realidad social difería notablemente en relación con el desarrollo europeo donde la media de analfabetismo se establecía en esos años en el $25 \%$ de la población. Asimismo, a pesar del crecimiento demográfico español, de los diecisiete millones de habitantes se contaría con un total de al menos cinco millones de ciudadanos como posibles lectores, de los cuales con formación superior llegarían a ser más de ciento sesenta mil personas, es decir, el 1\% de la población (Núñez, 1998: 245-246). En consecuencia, el público lector es realmente reducido a la vez que resulta una representación limitada de la sociedad y de aquella parte de la misma que pudiera interesarse, asimilar y debatir sobre las múltiples repercusiones del progreso industrial, los cambios políticos o la actualidad internacional.

Al analizar la situación de la prensa madrileña en 1898 también es preciso tener en cuenta que "a pesar de haber atravesado algunos momentos de crisis era ya una prensa consolidada, moderna, preocupada por todo tipo de temas y capaz de satisfacer las necesidades de información, formación y entretenimiento de sus lectores" (Saiz, 1998: 200). Entre los tres diarios seleccionados para este estudio, identificados como diarios noticieros y formando parte de las primeras cabeceras de la prensa española en cuanto al volumen de ventas, destaca El Imparcial puesto que contabilizó aproximadamente ciento treinta mil ejemplares en su tirada diaria de 1898 (Fuentes y Fernández, 1998: 164).

Con relación al estilo periodístico de los diarios en cuestión, en el caso de $E l$ Imparcial hay que considerar que "aunque los contenidos de sus páginas eran habitualmente menos objetivos que los de La Correspondencia [de España], tenía en conjunto más calidad para los lectores y se vendía muy bien" (Edo, 1998: 43). El diario La Correspondencia de España se denominaba "familiarmente "El gorro de dormir de todos los españoles", por ser diario de la noche y de gran circulación" (Olmedo, 2004: 193). Y en ese desarrollo inicial del negocio empresarial emprendido por los tres diarios, donde priman los contenidos informativos y también de entrete- 
nimiento, El Liberal, que provenía de la escisión de El Imparcial por su sector más radical defendiendo una ideología republicana moderada, se había convertido en su principal rival y, con gran acogida entre el público lector, destacaba por sus crónicas, entrevistas y reportajes.

La actividad periodística de los diarios La Correspondencia de España, El Liberal y El Imparcial se vinculaba a ese incipiente periodismo de empresa, no adscrito tanto a las directrices de un partido político o sindicato, y que se conceptuaba como un servicio informativo con el fin de tratar cuestiones de interés público y ofrecer contenidos en relación con las predilecciones y gustos personales de sus lectores gracias a la labor realizada por intelectuales y periodistas. Su principal objetivo era aumentar el número de lectores, razón fundamental para justificar su competencia, y su propósito era dirigirse a "un profesional medio, agobiado por su trabajo y más predispuesto a olvidar en su rato de ocio los sinsabores de la vida laboral que a interesarse por los grandes problemas. Reacciona más fácilmente a los estímulos emotivos que a los elocutivos" (Núñez, 1998: 257). Por tanto, el tratamiento informativo de los tres diarios estaría condicionado a la considerada alta probabilidad de que el lector no pretendiese realizar una lectura pormenorizada, atenta y reflexiva en el escaso tiempo del que disponía a lo largo del día.

En cuanto al año 1898 asociado al estado del caso Dreyfus, por una parte consideramos que el deterioro de la imagen internacional de Francia generó un circo mediático transnacional en el que participaron los múltiples actores de la vida social: jueces, ministros, el ejército, ciudadanos, medios de comunicación, artistas e intelectuales. Con relación a España, a pesar de ser una época realmente convulsa con el fin del Imperio español (Serrano, 1984), existió un interés por informar de la evolución del asunto Dreyfus, siendo durante ese año "el suceso más comentado y que se mantendría en las páginas de los periódicos" (Armero, 1998: 21). Asimismo, en los diarios analizados se procuró comprender la reacción de la sociedad francesa puesto que "muchos intelectuales, economistas o políticos del siglo XIX consideraron que vivían en un mundo de redes" (Rueda, Galán y Rubio, 2014: 39-40) y, por consiguiente, se puede observar la existencia de un sentimiento de pertenencia a la comunidad internacional y que las consecuencias del caso Dreyfus y la situación de Francia lograsen una implicación por encima de sus propias fronteras. La primera hipótesis del presente artículo hace referencia a cómo se desarrolló la cobertura informativa y, asimismo, cómo se llevó a cabo el tratamiento de la información en los diarios madrileños La Correspondencia de España, El Liberal y El Imparcial durante 1898. Nos hemos planteado si uno de los tres diarios difundió un mayor número de contenidos periodísticos y con una frecuencia de publicación superior. Si tal vez se desarrolló una comunicación informativa de los hechos o si se llegó a valorar una evolución de los acontecimientos. Si contaron con la participación de los intelectuales de la época y si se emplearía como un tema de interés social.

El inicio del caso Dreyfus se establece el día 22 de diciembre de 1894, cuando el consejo de guerra encargado de juzgar al capitán Dreyfus declara su condena por alta traición, y concluye el 12 de julio de 1906, día en el que el Tribunal de Casación reconoce la inocencia de Dreyfus y su rehabilitación completa en el ejército. Un arduo proceso judicial en el que tiene lugar, por encima de la carga violenta y el imponente peso ideológico de los múltiples sucesos públicos, la confrontación de "la Justicia y la Verdad alzadas frente a la intolerancia y la razón de Estado" (Peter, 1961: 1163). Por consiguiente, no sólo se evidencia la crisis de Francia y la insolvencia de sus 
instituciones a través de este suceso sino que se expresa una lucha de ideales determinante en la movilización de todos los agentes sociales.

Desde el momento en que el capitán Dreyfus fue detenido se emplearía como un asunto público dado que la prensa francesa, diarios como L'Éclair, Le Figaro, Le Soir, y la agencia Havas se encargaron de comunicar, el mismo 1 de noviembre de 1894, breves informaciones que anunciaban un caso de espionaje, con connotaciones xenófobas y antisemitas más evidentes en aquellos diarios que se posicionarían como «antidreyfusistas» siendo ejemplo de ello La Libre Parole (Caballos, 2002: 42-43). Se llevaría a cabo un seguimiento periodístico de la constante evolución del caso, de las reacciones que suscita y del ambiente de desasosiego y de expectación (Denis, Lagrée y Veillard, 1995). A finales de 1897 se constata que: "La discordia a favor y en contra de Dreyfus continuó con un salvajismo cada vez mayor. Comprendió al conjunto de los actores políticos y extendió el caos a todas las áreas de la vida privada." (Steevens, 1899: 10). En el periodo seleccionado para este estudio tienen lugar numerosos acontecimientos clave para el proceso. No solo se juzga al comandante Esterhazy, al coronel Picquart o al comandante Henry, sino también a Émile Zola.

El ámbito de la política había sido cuestionado por Émile Zola en sus publicaciones años anteriores, del mismo modo que aquellos sucesos de actualidad que connotasen un cierto debate ético, a fin de provocar algún impacto en sus lectores y favorecer la posible reflexión y el diálogo social. Por consiguiente, acorde con sus principios morales y a su obra, decidió implicarse públicamente en defensa de la inocencia de Dreyfus y redactó su polémico «J'accuse» el 13 de enero de 1898 en el diario L'Aurore (Girard, 1955: 528). Como consecuencia directa, en el mes de febrero Zola sería condenado por difamación hacia los oficiales y diferentes personalidades a los que aludía en el escrito y, tras el recurso de la sentencia, le condenaron por rebeldía al haberse exiliado a Inglaterra a pesar de que hubiera considerado que su vida estaba en peligro tras recibir amenazas de muerte (Zola, 2004: 10). Tanto su misiva «J'accuse» como su condena cambiarían su vida al convertirse en un actor clave del caso Dreyfus. Suscitaría un mayor interés y responsabilidad ciudadana a nivel nacional e internacional y las principales cabeceras periodísticas así lo manifestaron. Sin embargo, la intervención de Émile Zola a favor de la impunidad del capitán Dreyfus fue entendida también como la posibilidad en aquel momento de "lanzarse en el combate cívico de Zola y de apropiarse de una soberanía completamente democrática donde un ciudadano había ido en defensa de otro ciudadano al ser perseguido por cuestiones de raza y de religión" (Duclert, 2010: 74).

En el transcurso del año 1898 la actividad de la prensa francesa e internacional es muy significativa respecto a la valoración sobre el denominado "caso Dreyfus". Las manifestaciones y disturbios antisemitas no cesan. La inestabilidad del Ministerio de Guerra provoca mayor agitación en Francia. Y, entre las múltiples respuestas, la Generación del 98 se posiciona públicamente en defensa de la inocencia de Dreyfus y la libertad de Zola. Una negligencia judicial, cuestionada públicamente que trascendió en una aguda situación de crisis, se expresaría en los diarios con gran preocupación y, como afirmaba Eusebio Blasco Soler, "Sin duda ninguna que es el principio de una guerra, no sé si civil o europea; pero lo es. (...) Y el proceso Dreyfus ha venido a ser el estallido nacional, la explosión que viene a amenazar seriamente la paz, poniendo a Francia, al país cerebro de Europa, como ellos dicen, al nivel de los pueblos menos civilizados"2.

El Liberal, 16-III-1898, «Triste reacción», p. 1. 
El siguiente estudio tiene por objeto determinar el empleo de los diarios citados como espacio informativo para conocer la actualidad del caso Dreyfus. Analizaremos la tirada anual, la frecuencia de publicación, el orden de los contenidos, el posicionamiento y la extensión de los textos, el lenguaje empleado, el tipo de redacción y las fuentes de información. La muestra será acorde a los momentos más relevantes del seguimiento periodístico que fue llevado a cabo en 1898. Un primer estudio de la cobertura informativa comprende el análisis de los 1095 números publicados. Posteriormente, se han recopilado aquellos ejemplares que aportaban explícitamente información relativa al caso Dreyfus y, como resultado, hemos obtenido 151 ejemplares de La Correspondencia de España, 135 ejemplares de El Imparcial, 170 ejemplares de El Liberal. Un total de 456 ejemplares componen la muestra para el análisis cuantitativo y cualitativo del tratamiento periodístico, lo que supone el $42 \%$ de la tirada anual. El porcentaje de ejemplares desestimados corresponde a las publicaciones en las que no se menciona el tema tratado y un $0,9 \%$ de ejemplares ilegibles debido a la deficiente conservación.

\section{Seguimiento periodístico durante el año 1898}

En primer lugar, El Liberal dedicó el 46,57\% de su tirada anual. Seguido del 41,37\% de La Correspondencia de España, se sitúa finalmente El Imparcial con el 36,98\%. Asimismo, se distinguen dos etapas de mayor cobertura informativa que corresponden a los meses de enero a febrero y de septiembre a octubre. En el primer periodo, los tres diarios proporcionaron de forma detallada y continuada determinados avances en relación con la comparecencia de Esterhazy ante el Consejo de guerra, la publicación «J'accuse» de Zola en el diario L'Aurore o la multitud de manifestaciones antisemitas acontecidas por toda Francia y en Argelia. Asimismo, febrero se convirtió en el mes de mayor seguimiento informativo realizado por los diarios en cuestión debido al desarrollo del proceso judicial contra Émile Zola. En este contexto, es preciso destacar que La Correspondencia de España destinó un total de veinticuatro números en enero y veintiséis en febrero. La información se publicó cada cuatro días a principios de enero y, a partir del día 9, la labor periodística se intensificó a través de una difusión diaria. En febrero se mantuvo esa misma constancia, excepto el día 2, en cada uno de los veintisiete días restantes del mes. Como resultado, La Correspondencia de España fue el diario que más ejemplares editó en los meses de enero y febrero de 1898.

En cuanto a El Liberal, se publicaron veintiún y veinticuatro ejemplares respectivamente. En la primera mitad de enero, la periodicidad de su información resulta irregular, dado que sólo encontramos referencias puntuales entre los días del 4 al 6 y del día 11 al 13. No obstante, a partir del día 15 de enero hasta el último día del mes, se estableció la difusión diaria de contenidos. En febrero se incrementó de un modo notablemente regular, entre los días del 3 al 5 de febrero y, a partir del día 7, se comunicó diariamente la evolución del proceso abierto contra Zola y de los múltiples sucesos antisemitas. La actividad periodística de El Liberal resultó muy similar a la de El Imparcial en cuanto a número de ejemplares publicados. Con un total de diecinueve ejemplares en el mes de enero y veinticuatro en febrero, la frecuencia de publicación de El Imparcial fue diaria entre los días 11 al 14 de enero y, posteriormente, a partir del día 18 hasta finales de mes. En febrero de 1898 la periodicidad fue constante durante todo el mes, con intervalos de máximo un día sin publicar ninguna información. 
Los temas tratados por la prensa en septiembre y octubre de 1898 comprenden la crisis ministerial de Francia, el suicidio del teniente coronel Henry, el procesamiento del coronel Picquart, la solicitud de revisión del juicio a Dreyfus, la correspondencia de la familia Dreyfus y algunos disturbios antirrevisionistas ocurridos en París. Asimismo, los diarios españoles participaron en la difusión informativa proveniente de la prensa francesa, inglesa, italiana y alemana. A finales de octubre se analizó en profundidad si el Tribunal Supremo determinaría que se procediera a la revisión del caso y, aunque Dreyfus seguiría en la Isla del Diablo, este hecho supuso un cambio decisivo ante la gran incertidumbre que se vivía en las semanas precedentes. La Correspondencia de España alcanzó la publicación de veintiún ejemplares en septiembre y diecinueve en el mes de octubre. Resultó menos constante que en los primeros meses del año y, por consiguiente, encontramos intervalos de máximo tres días sin informar junto con la presencia diaria de contenidos en las semanas del 5, 19 y 26 de septiembre.

El diario que llevó a cabo la actividad informativa más intensa de todo el año fue El Liberal. En septiembre destinó veinticinco ejemplares y, en octubre, veintiséis. Entre los días 1 y 11 de septiembre se informó de forma constante, después se publicó puntualmente el día 14, y se reanudó la comunicación diaria de contenidos desde el día 18 hasta finales de mes. En octubre, mes de mayor difusión para el periódico, encontramos un primer intervalo de publicación diaria, del día 1 al 16. Se prosiguió de forma puntual, los días 19 y 20, para continuar sin cese alguno hasta concluir el mes. En relación a El Imparcial, el periódico dedicó veinte ejemplares en septiembre. Llevó a cabo una publicación diaria los primeros siete días del mes, después se contabilizan informaciones puntuales cada dos días a mitad de mes y, a continuación, se retomó la transmisión diaria de noticias a partir del 22 de septiembre. El mes de octubre resultó más irregular, con tan solo diecisiete ejemplares y un intervalo de dos a cuatro días entre cada contenido publicado. La frecuencia de la última semana del mes es diaria debido a la relevancia de la revisión del proceso judicial contra Dreyfus.

Seguimiento informativo durante 1898
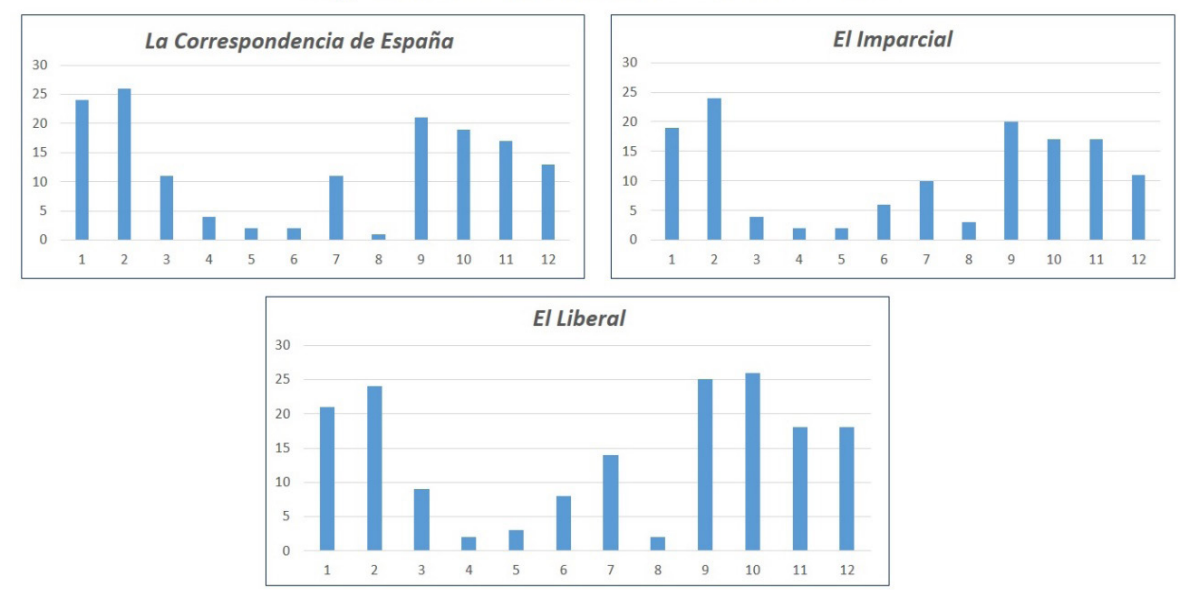

Tabla 1. Evolución del seguimiento informativo realizado por los diarios La Correspondencia de España, El Imparcial y El Liberal en 1898 sobre el caso Dreyfus (Elaboración propia). 


\section{Ubicación de la información en cada periódico}

El tratamiento de la información que realizan los diarios también comprende las variables relacionadas con el tamaño de los textos periodísticos y su distribución dentro del orden de contenidos. Con una extensión común de cuatro páginas, destinando la última a los anuncios publicitarios, los diarios El Liberal y La Correspondencia de España poseían un formato a seis columnas. Caso contrario es El Imparcial, que se estructuraba en cinco. Un aspecto común en los tres es el hecho de delimitar el espacio de cada página a través de líneas verticales para marcar las columnas y del empleo de líneas horizontales para diferenciar los textos periodísticos y las secciones. Asimismo, se modifica la tipografía de cada encabezamiento de la pieza periodística para destacar el titular de una noticia puntual o el nombre de la sección de un determinado espacio informativo. Otro elemento que altera la división de las informaciones es la difusión de novelas de folletín en La Correspondencia de España y en El Imparcial. Asimismo, la distribución de los contenidos dependía de la relevancia de la noticia dentro de la temática internacional, dado que se comunicó la actualidad de las guerras en Cuba, en Filipinas, en Manila, el hundimiento del Maine, las acciones de Bismarck o el conflicto de Fashoda.

A lo largo del año, en 151 ejemplares de La Correspondencia de España se hizo referencia al caso Dreyfus. En seis ocasiones encontramos un máximo de dos informaciones en diferentes secciones de un mismo ejemplar, incluso en páginas alternas, de modo que el número total asciende a 157 informaciones publicadas. Asimismo, se otorgó un espacio específico en el periódico y, como resultado, los lectores localizarían las noticias de forma habitual en la página 2 del diario, es decir, en setenta y seis ocasiones. El segundo lugar para este tipo de información fue la página 3, contabilizada sesenta y siete veces. De un modo menos frecuente, se publicó en la primera página en nueve ocasiones; y entre las páginas 2 y 3 en un total de cinco. $\mathrm{Su}$ ubicación también variaría según la extensión de la noticia y del espacio disponible, aunque se tiende a publicar la información completa en una sola página frente a la posibilidad de comenzar en la página 2 y concluir en la página 3 del diario.

En El Liberal se publicó más de un texto periodístico el mismo día y, en un total de veintisiete ejemplares, se redactaron de una a dos piezas informativas en diferentes secciones de las páginas 1, 2 y 3 para profundizar en aspectos concretos del caso Dreyfus. Esta variación resulta significativa para el estudio porque comprende el $15,88 \%$ del total de ejemplares. Por consiguiente, se han identificado 170 números publicados donde se comunicaron la suma de 192 contenidos periodísticos. El primer espacio de difusión fue la página 1 del diario, en un total de ochenta y ocho ocasiones, frente a la ubicación en la página 2 , que son setenta y seis. Se recurrió al empleo de la página 3 veinticinco veces. De forma puntual, hemos encontrado un caso en el que se ha publicado en la página 4 del diario, correspondiente al día 14 de noviembre de $1898^{3}$. En tan solo dos publicaciones la información tiene inicio en la página 1 y concluye en la página 2 , debido a la distribución de la gran diversidad de información publicada.

Una organización muy similar desarrolló El Imparcial porque predomina el posicionamiento de los contenidos en la primera página, en un total de sesenta y siete

El 14-XI-1898 El Liberal informó exclusivamente sobre la recepción pública de Isidoro Fernández Flórez, junto con el discurso de Juan Valera, en las dos primeras páginas. 
ejemplares. La disposición de la información en la página 2 también resulta recurrente, en cincuenta y tres, y se empleó puntualmente la página 3, en tan sólo trece ocasiones. Asimismo, en cuatro números del diario encontramos que se ha optado por iniciar la pieza periodística en la página 1 y su término se amplió hasta la página 2. Como resultado, El Imparcial proporcionó a sus lectores un total de 137 contenidos periodísticos en sus 135 números publicados. Es preciso destacar la gran extensión del espacio destinado para analizar la evolución del caso Dreyfus, se llegó a superar la redacción de una columna completa y con prioridad en las partes superior y central de la página.

Posicionamiento de la información

\begin{tabular}{|l|c|c|c|}
\hline $\begin{array}{l}\text { Ubicación de los textos } \\
\text { publicados }\end{array}$ & $\begin{array}{c}\text { La Correspondencia de } \\
\text { España }\end{array}$ & El Imparcial & El Liberal \\
\hline Página 1 & 9 & 67 & 88 \\
\hline Página 1-2 & - & 4 & 2 \\
\hline Página 2 & 76 & 53 & 76 \\
\hline Página 2-3 & 5 & - & - \\
\hline Página 3 & 67 & 13 & 25 \\
\hline Página 3-4 & - & - & - \\
\hline Página 4 & - & - & 1 \\
\hline TOTAL DE TEXTOS PUBLICADOS & 157 & 137 & 192 \\
\hline
\end{tabular}

Tabla 2. Análisis del posicionamiento de las informaciones publicadas sobre el caso Dreyfus en 1898 por los diarios La Correspondencia de España, El Imparcial y El Liberal (Elaboración propia).

\section{Evolución de los titulares}

Los diarios variaron sus titulares según el transcurso de los acontecimientos. Como características comunes, la tipografía era de mayor tamaño que la aplicada habitualmente y, sobre todo, en relación con el texto de la noticia. Asimismo, solían ser muy breves, en su mayoría compuestos por cuatro palabras, y llamativos sin perder su valor explicativo. Como resultado, el lector identificaría una serie de secciones de publicación periódica, exclusivas para este tipo de información, y encontraría con gran facilidad los últimos sucesos más actuales. El principal titular de La Correspondencia de España fue «La cuestión Dreyfus», presente en treinta y un textos periodísticos. Se utilizó en los meses de enero, septiembre y octubre; en menor medida, también aparece en noviembre y diciembre. El segundo titular más empleado fue «Zola y Dreyfus» en veintiuna ocasiones correspondientes a los contenidos publicados en enero y febrero. Otra variante, «Zola ante el jurado», del mes de febrero, aparece en diecisiete informaciones. Se constata la existencia de una serie de secciones fijas y se recurrió a la repetición de titulares de forma puntual como en «Zola sentenciado», «En honor a Zola», «Madame Dreyfus», «El proceso Dreyfus-Esterhazy», «La cuestión Zola»o «Un testigo excepcional». 
El uso de un lenguaje más transgresor por parte de El Liberal queda reflejado en la elaboración de los contenidos. El titular más frecuente fue «El escándalo Dreyfus», empleado en un total de sesenta y una ocasiones entre los meses de enero, julio, septiembre, octubre y diciembre. El segundo más utilizado fue «El asunto Dreyfus», en veintiocho textos periodísticos de noviembre y diciembre. En diecinueve ocasiones se aplicó «El proceso Zola» durante febrero, mayo y julio. Asimismo, El Liberal presentó una mayor variedad de contenidos. En consecuencia, se nombra a Zola explícitamente, como en «Zola supersticioso» en el mes de enero, «Zola absuelto» publicado en abril, o «Zola triunfa» en septiembre. En este sentido, también apareció explícitamente Dreyfus en titulares de informaciones puntuales como en «La cuestión Dreyfus», que encontramos una vez en enero y otra en noviembre; o «Los secretos de la cuestión Dreyfus» en octubre. Otro ejemplo es «El proceso Dreyfus», utilizado en cinco informaciones entre julio, agosto y noviembre, y «La revisión del proceso Dreyfus» de septiembre.

En el diario El Imparcial se crearon secciones temporales en el 76,8\% de sus ejemplares. Predominó el empleo de «La cuestión Dreyfus», en veinticinco ocasiones, principalmente en enero y febrero. El titular «El asunto Dreyfus» apareció como una opción del anterior, utilizado en siete informaciones en los meses de julio, septiembre y octubre. Asimismo, encontramos en el mes de febrero «Zola ante los tribunales», repitiéndose en mayo, y se utilizó en un total de quince textos periodísticos. Desde septiembre hasta diciembre son muy frecuentes «El proceso Dreyfus», en diecisiete ocasiones, y «La revisión del proceso Dreyfus», presente en trece informaciones. En El Imparcial también se analizó la situación de Emile Zola. En febrero, se publicaron quince textos titulados «Zola ante los tribunales». Otros tres textos en el mes de julio, cuyo titular fue «La fuga de Zola», y otros contenidos puntuales se editaron a lo largo del año con titulares como «Zola fugitivo, Zola exonerado», «Zola y la Legión de Honor», «Barbier y Zola», «El coronel Echagüe y Zola», «Zola y sus detractores».

Desde los tres diarios se comunicó la crisis ministerial de Francia con relación al caso Dreyfus. Cada periódico proporcionó una media de ocho a doce textos en los meses de junio y octubre. En La Correspondencia de España se emplearon titulares como «Crisis Ministerial» y «Conjura militar». En El Liberal se hizo referencia a este tipo de información mediante «La crisis francesa» y «Conspiración militar en Francia». Un modelo similar empleó El Imparcial con «La crisis en Francia», «Francia sin ministerio»y «La conjura en Francia». Siguiendo el mismo tratamiento informativo, los diarios trataron en cinco ocasiones las manifestaciones y disturbios antisemitas, que comprendieron el territorio francés y parte del extranjero, en los meses de enero, febrero, noviembre y diciembre. La Correspondencia de España comunicó los hechos a través de los titulares "Crisis en Francia» y «Alborotos en París». En el caso de El Liberal, encontramos «Agitación en Francia» y «Crisis francesa». El Imparcial recurrió a «Agitación antisemita» y «Disturbios por Dreyfus».

\section{Principales fuentes de información}

A través de la labor de sus corresponsales Argus y Huertas, La Correspondencia de España aportó informaciones actuales, similares a una crónica o reportaje interpretativo, con un breve titular como «Zola y Dreyfus» o «Zola ante el jurado». Siempre 
con la referencia «Por telégrafo. De nuestro corresponsal particular», se publicaban con un día de demora, con firma y se precisaba el lugar, el día y la hora de la redacción. Se transmitían los detalles del ambiente y se reproducían de forma explícita los diálogos y declaraciones. Podemos apreciar un intento por trasladar la realidad de los hechos y, en situaciones de gran confusión, se explicaban multitud de detalles a los lectores, por ejemplo: «Trescientos estudiantes recorrieron las calles de Nancy, gritando: “¡Mueran los judíos! ¡Abajo Zola!”. En todas las ciudades se hicieron prisiones. - Huertas $\rangle^{4}$. En cuanto a las agencias de noticias, La Correspondencia de España recurrió a Fabra. Sus contenidos se indicaron bajo el epígrafe «De la agencia Fabra» dentro de la pieza informativa. No obstante, en su mayoría se ubicaron en «Extranjero», sección redactada habitualmente en la página 3. Con un retraso de uno a dos días en su difusión, aparecen con un subtítulo explicativo introductorio, la indicación de la ciudad y el día, un texto muy conciso y el nombre de la agencia como cierre.

En las informaciones que se recibían por telégrafo en El Liberal no consta una firma de corresponsal y, por el contrario, se precisa la firma de las agencias Fabra y Havas después del cuerpo de la noticia. La presentación de estos contenidos comprendía un titular y un subtítulo de gran tamaño, en menor tipografía "Por telégrafo» o «Telegramas de nuestro servicio particular» y se detallaban la ciudad, el día y la hora. En la sección «El telégrafo. Servicio particular de El Liberal» se estableció un apartado específico denominado «De la agencia Fabra», con contenidos muy concisos y puntualizando el país, la ciudad y el día. También se dispuso de la sección «Exterior» para trasladar a los lectores los sucesos de ámbito internacional, así como las novedades sobre Dreyfus y la crisis ministerial francesa, proporcionados por las agencias Fabra y Havas. De forma excepcional, encontramos en febrero un texto firmado por Aguilar, recibido por telégrafo desde Valencia, para narrar la muestra de apoyo y felicitación a Zola desde El Pueblo ${ }^{5}$. Otra de las fuentes de información fue la correspondencia del mismo Alfredo Dreyfus a su esposa durante sus años en la Isla del Diablo. De septiembre a noviembre de $1898, E l$ Liberal editó «Las cartas de Dreyfus» ${ }^{6}$, en la página dos, respetando la integridad de la redacción.

En El Imparcial dispusieron de un corresponsal en París, L. Arzubialde, para dar continuidad al seguimiento periodístico durante todo el año a través de las secciones «Desde París», «La cuestión Dreyfus» ${ }^{7}$ o $« E l$ proceso Dreyfus». Con la indicación «Por telégrafo. De nuestro corresponsal» y con la firma «A.» o «Arzubialde», como variante en extenso, su publicación contaría con un retraso de máximo un día. Arzubialde también cubrió sucesos puntuales, como «Picquart y Esterhazy» en marzo o «Fuga de Zola» en julio. Desde la sección «Servicio telegráfico de "El Imparcial"», se recogieron breves avances informativos, divididos en pequeños titulares, con la misma diferencia de un día de publicación, firmados siempre, detallando el lugar, el día y la hora exacta. En este espacio se distinguieron en dos apartados los contenidos «De nuestros corresponsales», como L. Arzubialde, frente a las noticias «De la agencia Fabra».

\footnotetext{
La Correspondencia de España, 19-I-1898, «La cuestión Dreyfus. Tumultos en Francia», p. 3

El Liberal, 17-II-1898, «Mensaje a Zola», p.2.

El Liberal, 21-IX-1898, «Las cartas de Dreyfus. Primera serie», p. 2.

El Imparcial, 28-XII-1898, «La cuestión Dreyfus», p. 2.
} 
Los tres diarios emplearon como fuente de información las principales cabeceras internacionales para transmitir la opinión pública internacional. De la prensa francesa, hicieron referencia a Le Figaro, Le Matin ${ }^{8}, L^{\prime}$ Éclair, L'Aurore, La Lanterne, Le Radical, Le Temps, La Fronde o Le Siècle. Asimismo, consideraron el análisis realizado por The Observer, New York Herald, Berliner Tageblatt, Daily News o Esercito Italiano. Otro de los recursos periodísticos fue la mención de determinados comunicados dirigidos a Zola por parte de la sociedad española. Publicaban el texto íntegro, incluso con el mismo titular. Y, en consecuencia, demostraron abiertamente su apoyo a la causa de Zola y que abogaban por la inocencia de Dreyfus. Ejemplo de ello es el «Mensaje a Zola», aviso que logró más de cuatrocientas firmas en tan solo un día y algunos de los signatarios fueron Jacinto Benavente, Alfredo Calderón, Valle Inclán, Vicente Casanova o Luís de Terán y Castillejo9. Otro ejemplo es «Una carta de Zola» ${ }^{10}$, misiva que Émile Zola envió a Miguel Sawa, director de Don Quijote, por el apoyo recibido de la juventud española.

Por último, es preciso mencionar el modo en que los propios diarios cuestionaron y valoraron los sucesos del caso Dreyfus y cómo colaboraron los intelectuales en la redacción de este tipo de contenidos. Desde La Correspondencia de España se publicaron de forma puntual algunos textos, a modo de editorial, en la página 1 del diario. En «Comentarios de la redacción», encontramos en enero «La cuestión Dreyfus» $\rangle^{11}$, escrito por G.A., donde se cuestionaba el grado de negligencia tolerado por el ministro de Justicia Sarrien y, en especial, la opinión de los periódicos alemanes. En el diario El Liberal la sección «Crónicas» se convirtió en el primer espacio para facilitar a los lectores contenidos de mayor carácter reflexivo e interpretativo. Un total de veinticuatro textos periodísticos, en primera página, con una extensión de mínimo una columna completa, concluían con la firma del autor y la fecha de redacción. Bajo el subtítulo «París», Luís Bonafoux expresó la valía literaria de Zola, la belleza de su acto de defensa a Dreyfus y a los principios de Francia. Bonafoux tomaba como ejemplo en sus comparaciones la obra de otros ilustres franceses, como Lamartine, Ernest Renan o Lucien Descaves.

Otro de los escritores asiduos en la sección cultural «Nuestros domingos» de $E l$ Liberal y que colaboró en la sección «Crónicas» fue Ernesto García Ladevese, analizando la gravedad de los actos antisemitas en «La raza proscrita» o en «Drumont». Asimismo, José Gutiérrez Abascal (Kasabal) mencionó en noviembre la repercusión social del caso Dreyfus en su escrito «Los guantes». Con una publicación más intensa en los meses de enero, febrero y marzo, cabe destacar que Joaquín Dicenta redactó « ¿La vida miserable...!!»; Luís Morote «Razas malditas» y «Ranciedades»; Eusebio Blasco «Triste reacción»; Alejandro Sawa «¿Por qué?», en alusión al sometimiento público de Dreyfus. En los citados textos se tomaron como referente la obra de Balzac, Víctor Hugo, Diderot, Voltaire, Cervantes y Shakespeare para recordar el legado cultural de Europa y alertar de que la razón y el civismo parecían corromperse frente a la degradación pública a Dreyfus, la violencia contra Zola y el sentimiento generalizado de odio hacia los judíos. Rafael Salillas manifestó en «El cuerpo extraño» ${ }^{12}$

\footnotetext{
El Liberal, 31-X-1898, «Una visita a la Isla del Diablo», p. 2.

El Imparcial, 12-II-1898, «En Madrid», p. 1.

La Correspondencia de España, 10-III-1898, «Una carta de Zola», p. 2.

La Correspondencia de España, 17-IX-1898, «Comentarios de la redacción. La cuestión Dreyfus», p. 1.

El Liberal, 25-I-1898, «Crónicas. El cuerpo extraño», p. 1.
} 
que la tolerancia religiosa era un convencionalismo europeo y los clichés eran reflejo de los odios y rencores históricos.

El diario El Imparcial desarrolló la sección «Gaceta de París», en la mayoría de los casos publicada en primera página, siempre con la firma de su corresponsal L. Arzubialde. Mediante reportajes y artículos de opinión se analizaron temas sobre la sociedad francesa, tanto del mundo de la cultura como hechos escabrosos y delictivos, y también sobre el caso Dreyfus aproximadamente una vez al mes. Con una diferencia de tres días desde su redacción, se aprecia una mayor carga interpretativa y se involucra directamente al lector mediante alusiones a su posible interpretación individual de los hechos. Por ejemplo, en septiembre L. Arzubialde muestra con claridad su opinión al explicar en el inicio de su crónica: «No creo necesario insistir sobre el efecto que habrá producido el coup de théatre ocurrido en el asunto Dreyfus, cuyos episodios revisten, cada vez más, todos los caracteres de un bien forjado melodrama» ${ }^{13}$. En la sección «Actualidades» del diario El Imparcial, José de la Serna (Gil Imón) escribió «Zola poeta» para comentar la simplicidad y nobleza de los inicios literarios de Zola, así como la relación de amistad del literato con Clemenceau. Otro de los artículos destacables es «Habla un judío español», por Mariano de Cavia, donde reprodujo ciertos versos del conocido «Don Sem Tob, Rabí de Carrión», del siglo XIV, para impulsar en los lectores su admiración hacia Zola. Mariano de Cavia expresó que «campeón es, en fin, Emilio Zola, no de la letra mortífera del Talmud, sino del espíritu vivificante de la libertad ${ }^{14}$.

\section{Conclusiones}

Los diarios españoles La Correspondencia de España, El Imparcial y El Liberal realizaron un seguimiento constante y exhaustivo de la noticia en relación con el desarrollo del caso Dreyfus. A lo largo del año 1898, La Correspondencia de España fue el diario que publicó un mayor número de contenidos informativos en el mes de febrero, momento en que tuvo lugar el proceso judicial contra Émile Zola. No obstante, El Liberal fue el diario que llevó a cabo una cobertura informativa más intensa, destacando la labor periodística del mes de octubre ante la posible revisión de la sentencia contra Dreyfus. En El Imparcial se dedicaron un menor número de ejemplares y contenidos publicados aunque se aprecia un alto interés por seguir la actualidad del caso Dreyfus en ese año.

El posicionamiento de la información, con prioridad entre la primera página y la segunda, muestra que el caso Dreyfus fue valorado por los diarios citados como un tema de gran relevancia, muy atractivo para su público lector, recurrente para informar sobre la actualidad internacional e, incluso, con la posible intencionalidad de pretender incrementar el número de lectores así como de ocupar el mismo lugar que correspondería a otros asuntos internacionales más conflictivos y de mayor significación para el interés nacional. La extensión de los contenidos publicados y de las secciones específicas sobre el caso Dreyfus permitiría encontrar con rapidez este tipo de contenidos, así como comunicar y sopesar los múltiples aspectos de los sucesos y de las reacciones de los diversos actores implicados.

El Imparcial, 03-IX-1898, «Gaceta de París. El proceso Dreyfus», p.1.

14 El Imparcial, 20-I-1898, «Habla un judío español», p. 1. 
Asimismo los tres diarios demostraron ser un espacio abierto a la participación de los intelectuales, de información y de reflexión sobre la complejidad de la realidad internacional de 1898.

La selección de los titulares realizada por cada diario denota el intento por captar la atención del lector y por facilitar la identificación de un mismo espacio informativo destinado para analizar y trasladar los acontecimientos relativos al asunto Dreyfus. El Liberal resultó emplear un lenguaje más provocador que también consta en sus titulares con el uso de «El escándalo Dreyfus», frente a «La cuestión Dreyfus» empleado por La Correspondencia de España y El Imparcial. Se caracterizan por su brevedad, concisión y causan una respuesta directa en el lector a fin de conocer también las reacciones de la sociedad francesa, de la prensa internacional y la repercusión en España.

El tratamiento informativo sobre el caso Dreyfus comprende tanto breves noticias, reportajes y crónicas como editoriales y artículos de opinión. Para ello, cada diario contó con sus propios corresponsales y redactores, con diversas fuentes de documentación y con la colaboración de intelectuales que se implicaron directamente a través de la prensa periódica redactando textos interpretativos y de opinión, saliendo de las secciones de cultura y sociedad y aportando mayor calidad a la cobertura informativa. Asimismo, La Correspondencia de España, El Imparcial y El Liberal recurrieron a la información proporcionada por las agencias de noticias Fabra y $\mathrm{Ha}$ vas, a las noticias publicadas en diarios internacionales, también a fuentes directas como testimonios, testificaciones y declaraciones públicas de las personas implicadas. Se constata una labor periodística por abordar todos los aspectos relacionados con el caso Dreyfus, por despertar a un lector que pudiera llegar a sentirse conmovido, indignado, abrumado o desconcertado. También se evidencia la determinación de cada uno de los diarios por comunicar y difundir, de un modo más significativo y cercano a la realidad, los mensajes redactados por los intelectuales de apoyo y compromiso con la causa.

\section{Referencias bibliográficas}

Armero, A. (1998). Fragmentos del 98. Prensa e información en el año del desastre. Madrid, España: Consejería de Educación y Cultura de la Comunidad de Madrid.

Barrera, C.; Sánchez, J. J. (1992). Historia del periodismo español desde sus orígenes hasta 1975. Pamplona, España: Ediciones Universidad de Navarra S.A.

Caballos, M. D. G. (2002). "El "affaire" Dreyfus: Un caso de xenofobia y antisemitismo en los albores del Siglo XX. Implicaciones políticas y literarias en la prensa francesa”. En Philologia Hispalensis. Revista de Estudios Lingüísticos y Literarios. Vol. XVI, Fasc. I. Sevilla, España: Universidad de Sevilla. pp. 37-71.

Chabás, J. (2001). Literatura española contemporánea, 1898-1950. Madrid, España: Verbum.

Denis, M.; Lagree, M.; Veillard, J. Y. (dirs.). (1995). L'Affaire Dreyfus et l'opinion publique en France et à l'étranger. Rennes, Francia: Presses universitaires de Rennes.

Duclert, V. (2010). "La República y el heroísmo democrático en el cambio de siglo" En Pagès, A. (ed.). Zola au Panthéon. L'épilogue de l'affaire Dreyfus. París, Francia: Presses Sorbonne Nouvelle. pp. 73-98.

Edo, C. (1998). "Los periódicos de Madrid en 1898”. En Estudios sobre el Mensaje Periodístico. No 4. Madrid, España: Servicio de Publicaciones UCM. pp. 39-60. 
Fuentes, J. F.; Fernández, J. (dirs.). (2010). Historia del periodismo español: prensa, política y opinión pública en la España contemporánea. Madrid, España: Editorial Síntesis.

- (dirs.). (1998, $1^{\text {a }}$ edición). Historia del periodismo español: prensa, política y opinión pública en la España contemporánea. Madrid, España: Editorial Síntesis.

Girard, M. (1955). "Positions politiques d'Émile Zola jusqu'à l'affaire Dreyfus". En Siegfried, A. (dir.). Revue française de science politique. $5^{\text {ème }}$ année. $\mathrm{N}^{\mathrm{o}} 3$. París, Francia: Presses Universitaires de France. pp. 503-528.

Núñez, L. (1998). "La prensa española en la crisis del 98”. En Alvira, R. et al. España, Estados Unidos y la crisis de 1898: Reflexiones para un centenario. Madrid, España: Fundación para el Análisis y los Estudios Sociales. pp. 245-283.

Olmedo, M. F. (2004). Anecdotario histórico español. Valencia, España: Carena Editors.

Peter, J. P. (1961). "Dimensions de l'Affaire Dreyfus". En Braudel, F. (dir.). Annales. Economies, sociétés, civilisations. $16^{\mathrm{ème}}$ année. $\mathrm{N}^{\mathrm{o}}$ 6. París, Francia: Armand Colin. pp. 11411167.

Rueda, J. C.; Galán, E.; Rubio, A. L. (2014). Historia de los medios de comunicación. Madrid, España: Alianza Editorial.

Saiz, M. D.; Seoane, M. C. (1998). Historia del periodismo en España. Vol. 3, El siglo XX: 1898-1936. Madrid, España: Alianza Editorial.

Saiz, M. D. (1998). “La prensa madrileña en torno a 1898”. En Historia y Comunicación Social. No 3. Madrid: Servicio de Publicaciones UCM. pp. 195-200.

Serrano, C. (1984). Final del Imperio. España, 1895-1898. Madrid, España: Siglo XXI.

Steevens, G. W. (1899). The tragedy of Dreyfus. Nueva York, Estados Unidos: Harper.

Tuñón, M. (2000) La España del siglo XIX. Vol. II. Madrid, España: Ediciones Akal.

Villacorta, F. (1980). Burguesía y cultura: Los intelectuales españoles en la sociedad liberal, 1808-1931. Madrid, España: Editorial Siglo XXI.

Zola, É. (2004). Yo acuso. Santa Fe, Argentina: El Cid Editor. 